\title{
A production train diagram of train control to save power consumption used for dynamic programming
}

\author{
T. Katori \& T. Izumi \\ Nihon University, Japan
}

\begin{abstract}
Reducing power consumption is an important subject for railways using electric energy. In order to efficiently use power generated by generation brakes, it is necessary to consider the acceleration or deceleration of trains running in each other's neighbourhood and control these factors as a whole. Therefore, we are studying to determine a train diagram that saves total and moment power consumption. However, because there are many combinations of how to run the trains, it is difficult to calculate the best solutions.

In this paper, we have solved the problem to create train diagrams to minimize power consumption considering the generation braking system. A computer uses conditions of distance between any stations and required running time for any number of trains, calculates how to run by dynamic programming in a solid model by time-distance planes, and shows S-curve train diagrams. Evaluation values are both total and moment power consumption. A characteristic function of power consumption is determined by train velocity of acceleration or deceleration. The solution is a combination how to run the trains minimizing the evaluation values.

Using this model, we calculated the appropriate schedule of train diagrams for the case of two trains running between five stations. The solution uses the generation braking system efficiently. For two trains, while one train increases its velocity, the other train reduces its velocity just at the same moment. Running conditions are able to combine gradient and limited high speed.
\end{abstract}

Keywords: trains control, power consumption, dynamic programming, train diagram. 


\section{Introduction}

For railway systems that are operated by electric power reducing power consumption is an important issue. There are many studies on how to save consumption in different departments, for example, dynamic train regulations, energy management, and so on. Generation brake systems, to effectively use power generated in the case of deceleration of the train, running near trains should be operated trains control considered acceleration and deceleration.

This study's aim is to determine the best schedule of train movement that has minimum total and moment power consumption, in the case of any number of trains running [1]. But because there are many combinations of how to run trains, it is difficult to determine the optimum solution. In this study field, studies exist that determine the running curves to reduce power consumption. These studies focus either on one train where the curve takes into consideration gradient or speed limitation, or they focus on any number of trains control where the form of the curve is held constant, and so on. However, in most studies, the former is for only one train though the running curve is complex, or the running curves form only a simple trapezium though the latter deal with any number of trains.

In this paper, we describe how to create a schedule of train diagrams by complex running curves for any number of trains to reduce the consumption generation brake for trains control. For this purpose we use the model of dynamic programming.

\section{Dynamic programming and model}

Dynamic programming (D.P.) is a method that works as follows: First, in order to reduce listing, many cases are integrated into one condition. Second, processes of listing solutions are such that they suit all conditions. Finally, states are dealt with abstractly transition, a best solution is caluculated[2].

\subsection{Running curve}

A running curve is a graph of time (or distance) versus speed when a train is running. A train can run any combinations of acceleration or deceleration, if it can reach the next station in a limited time. Figure 1 shows an example of a graph of time versus velocity. If the integrals of two running curves have an equal area each, the train can run both running curves in a limited time.

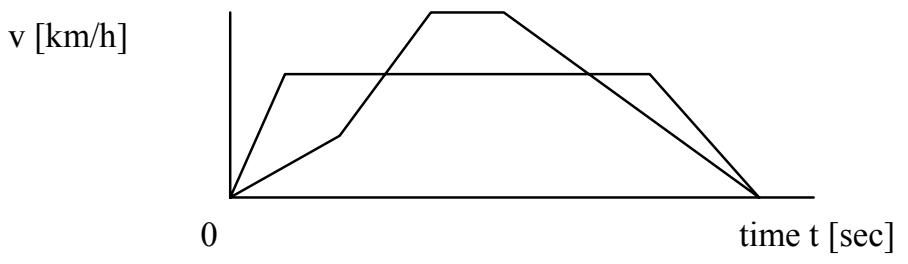

Figure 1: $\quad$ Sample of running curves. 
But the acceleration and also the power consumption of the motor differ from each other. To effectively use energy in the deceleration time by generation brake, there is a pattern of running curves for any number of trains that can reduce total and moment power consumption, adjustment acceleration and deceleration.

\subsection{For single train}

Figure 2 shows the concept of calculating the running curve to minimize the consumption using D.P. for a single train.

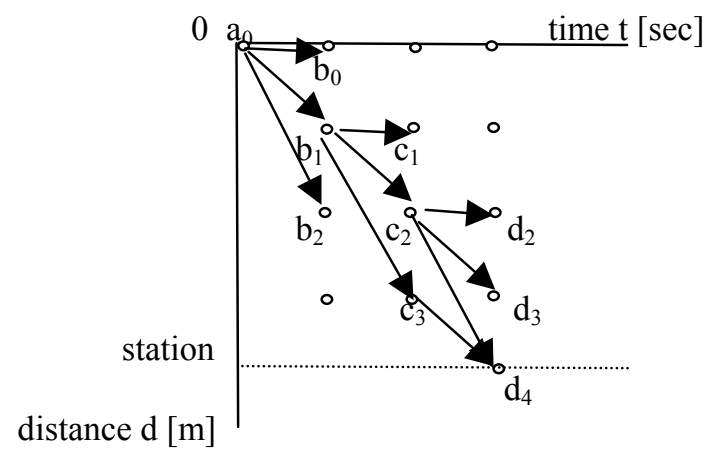

Figure 2: Calculation running curve for a single train using D.P.

Space in figure 2 is the same as the general train diagram. Gradient of an allow means velocity, and variation of the gradient allows means acceleration (deceleration). At each state (circles in figure 2), evaluation values (total and moment consumption) are calculated from $\mathrm{a}_{0}$ (time and location of origin station), and the values are replaced for a lesser one at same state. For example, in case of running to $d_{4}$ from $a_{0}$, there are 2 ways to reach $d_{4}$, namely one is " $a_{0}$ $\mathrm{b}_{1}-\mathrm{c}_{2}-\mathrm{d}_{4}$ ", the other is " $\mathrm{a}_{0}-\mathrm{b}_{1}-\mathrm{c}_{3}-\mathrm{d}_{4}$ ". The way that has lesser evaluation value at $\mathrm{d}_{4}$ is adopted.

\subsection{Expansion for over double trains}

Figure 3 shows how to expand this method for two trains. In this case, solutions of running curve are calculated in a three-dimensional plane composed of timedistance1 and time-distance2 space.

Evaluation values are both total and moment consumption.

$$
\begin{aligned}
& \mathrm{f}_{1}(\mathrm{i}, \mathrm{j}, \mathrm{t})=\min \left\{\mathrm{f}_{1}\left(\mathrm{~b}_{\mathrm{i}}, \mathrm{b}_{\mathrm{j}}, \mathrm{t}-1\right)+|\Sigma \mathrm{P}|\right\} \\
& \mathrm{f}_{2}(\mathrm{i}, \mathrm{j}, \mathrm{t})=\min \{|\Sigma \mathrm{P}|\}
\end{aligned}
$$

$f_{1}(i, j, t)$ : evaluation value of total consumption at time $t$, train 1 runs location $i$ and train 2 runs $j$.

$\Sigma \mathrm{P}$ : evaluation value of sum moment consumption by any number of trains at time $\mathrm{t}$. 


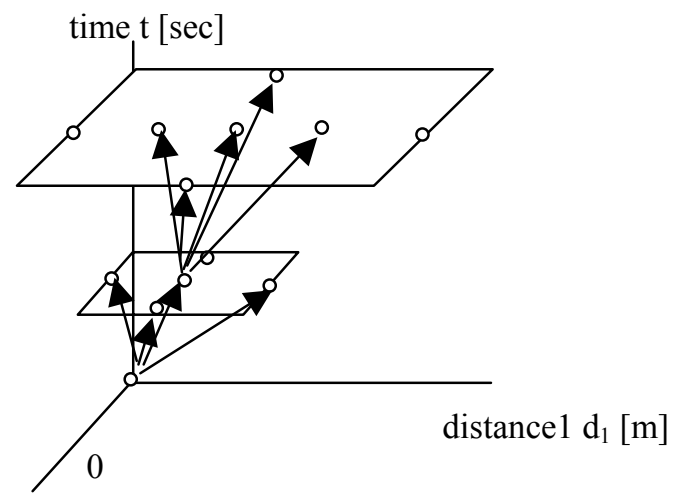

distance $2 \mathrm{~d}_{2}[\mathrm{~m}]$

(a) Calculating in the solution space.

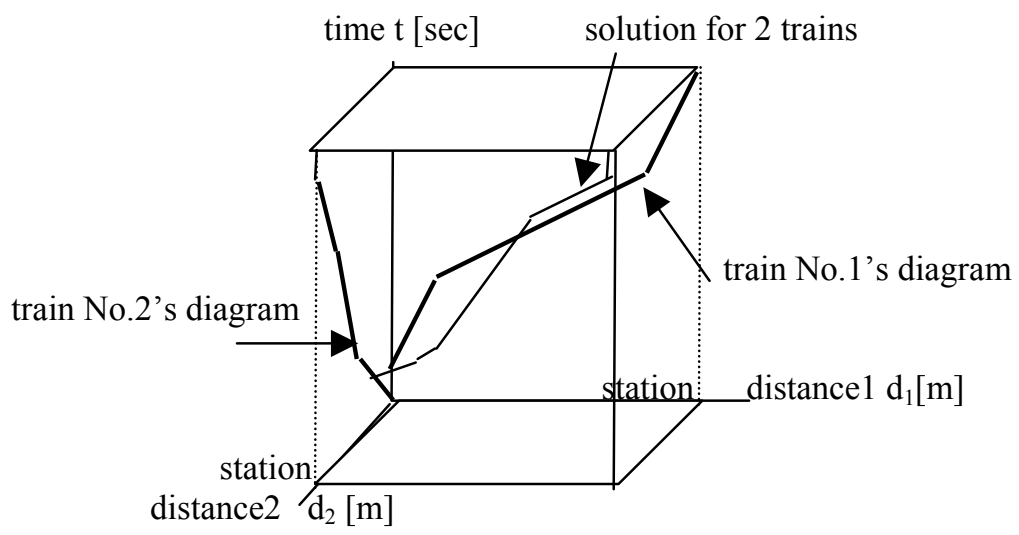

(b)An example of solution in the space.

Figure 3: Calculation running curves for 2 trains using D.P.

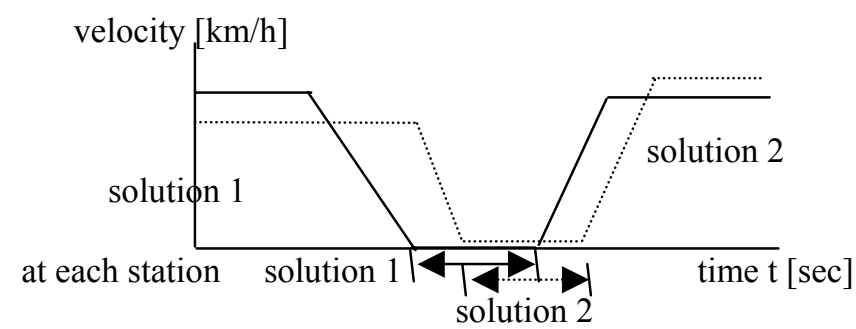

Figure 4: Keeping constant stopping time in solution calculation. 
At each time, both evaluation values $f_{1}$ and $f_{2}$ are replaced by a lesser value at the same state, and a train diagram of $\mathrm{S}$ curves is created as in figure 3 . If the solution projects to time-distancel plane, the curve means diagram of train 1 , and projects to time-distance 2 plane, means diagram of train 2 .

If there are some stations on the applied line, the solutions are calculated as such to keep constant stopping time at any stations, shown in figure 4 .

In the case of over 2 trains, the dimension expands to number of trains.

\section{Applying sample for virtual short line}

This method applies to short model lines. This model uses two lines.

(a) 5 stations in $1,600[\mathrm{~m}]$ per $400[\mathrm{~m}]$

(b) 5 stations in $1,900[\mathrm{~m}]$

Electric power is supplied by 1 substation.

Required time between stations is set, two trains follow each other. Stopping time at the station is 30 [sec].

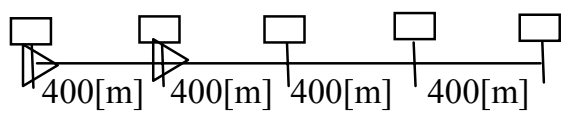

(a) 5 stations in $1,600[\mathrm{~m}]$

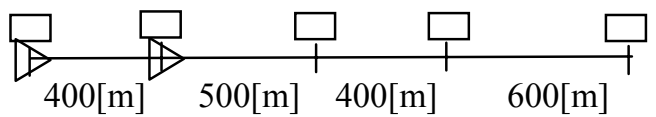

(b) 5 stations in $1,900[\mathrm{~m}]$

Figure 5: $\quad$ A sample short line. $(D$ : train $\square$ : station).

In this example we did not set running resistance or track gradient but it is possible to combine the conditions.

The current of acceleration consumption and deceleration generation [3] is a simple function determined only by velocity, this to show the effectiveness of the D.P. model that is presented in this paper. However, (for a more complete result) the location of substations or trains should be taken in consideration as well.

Figure 6 shows the current function.

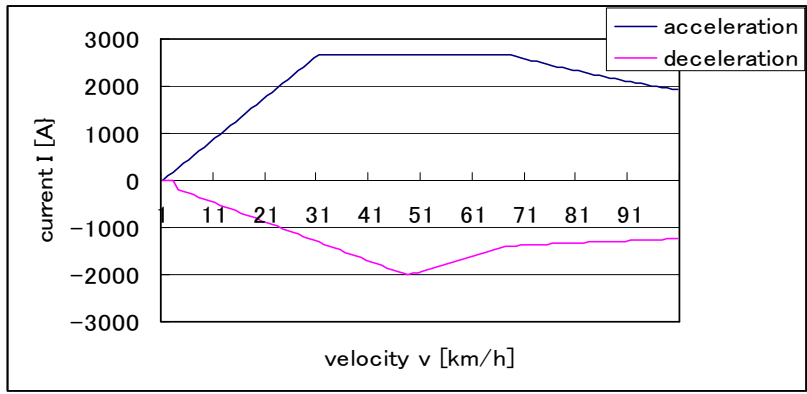

Figure 6: v-I characteristic curves. 
Catenary voltage also varies by pantograph location, but this study adopts a constant voltage of $1,500[\mathrm{~V}]$. Under these conditions, solutions of train operation that have minimum total and moment power consumption are shown in figures 7 and 8 .

\subsection{5 stations in $1,600[\mathrm{~m}]$ per $400[\mathrm{~m}]$}

The result of train operation is shown in figure 7 .

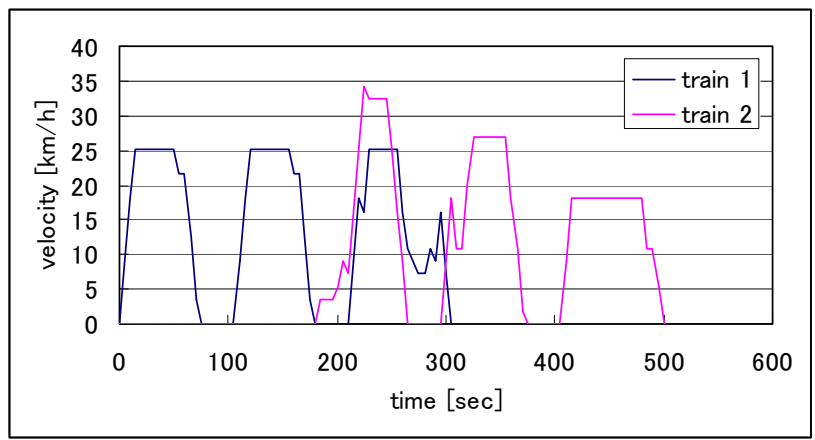

(a) Running curves.

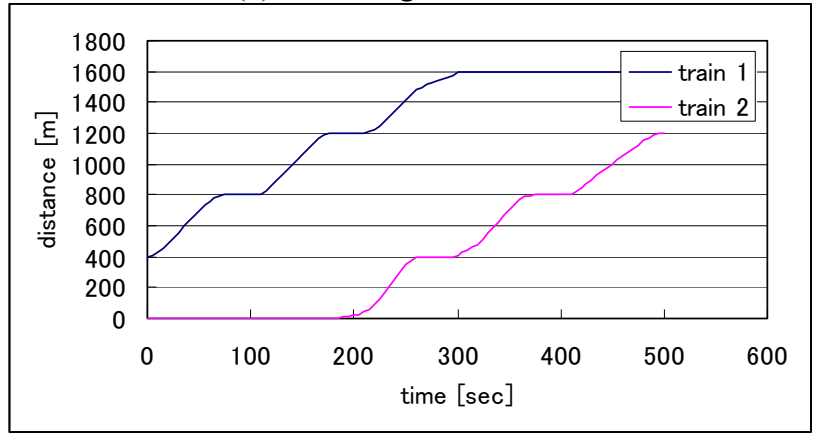

(b) Trains diagram.

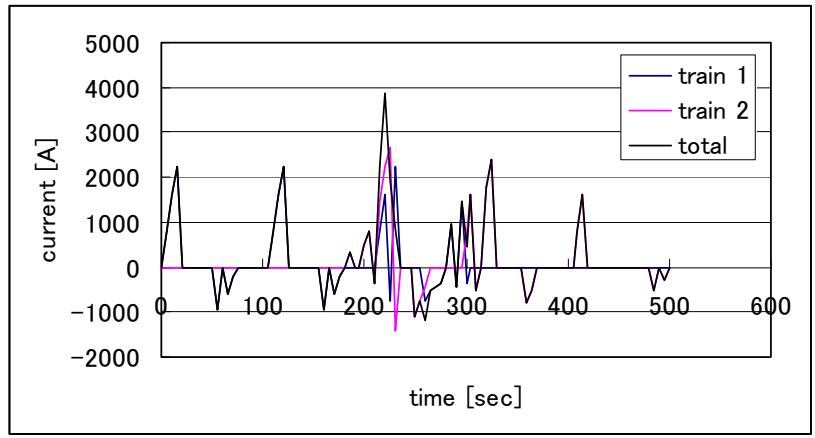

(c) Time characteristic for current.

Figure 7: Result of trains control to minimize power consumption for case (a). 


\subsection{5 stations in $1,900[\mathrm{~m}]$}

The result of train operation is shown in figure 8 .

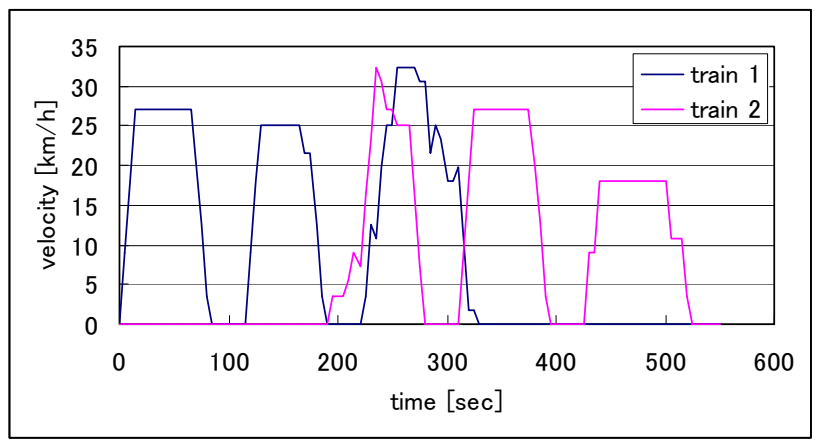

(a) Running curves.

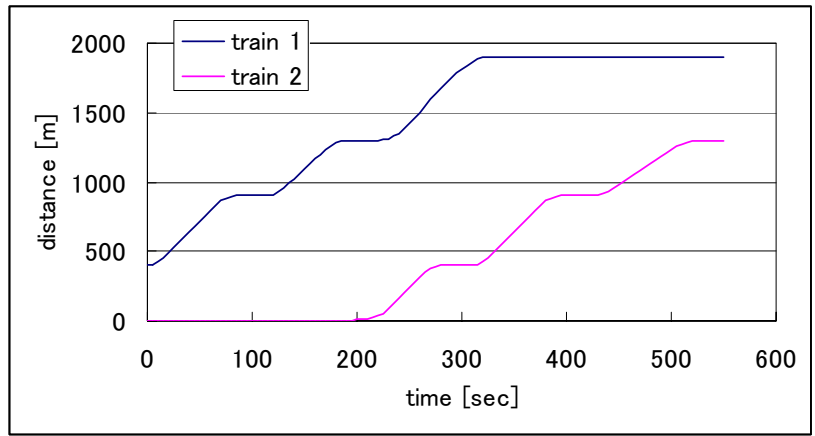

(b) Trains diagram.

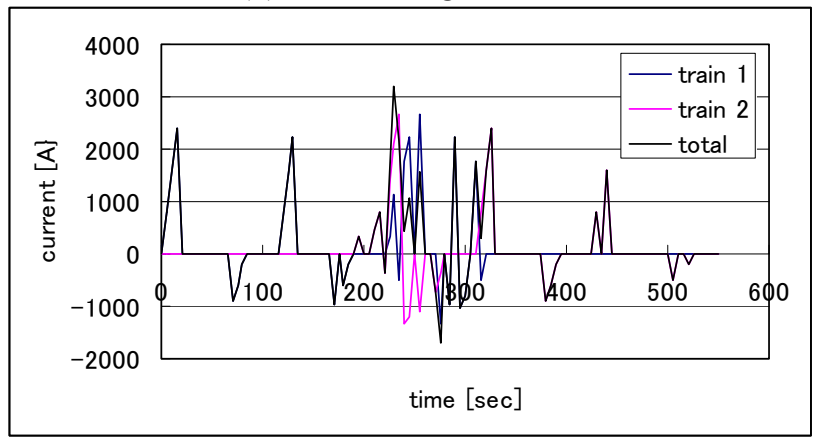

(c) Time characteristic for current.

Figure 8: Result of trains control to minimize power consumption for case (b).

In the case of only one train running, all running curves form a trapezium. These results are correct because generally the running curve of minimum consumption is said to be a trapezium form. 
Max speed in the solution is slow, because the distance between stations is short. However, generation brake are used effectively after 200 [sec] until 350 [sec] in figure 8(a), where one train accelerates and the other one decelerates at the same moment. After 50 until 150 [sec] and after 350[sec], loss of generation brake occurs when only one train is running. The rules to search solution that considered both lesser total and moment consumption cause the phenomenon.

Realistic running curves in short operation time can be expected, if weight is added to both evaluation values.

Figure 8 (b) expresses (a) for trains schedule, the scheduled diagram can reduce power consumption.

Figure 8 (c) shows moment current provided by (a). Table 1 shows total and maximal moment power consumption that the current multiplied catenary's voltage (D.C. 1,500[V] const.). Worst moment consumption that trains accelerate same moment expects $6.75[\mathrm{MW}$, but the consumption with $4.8[\mathrm{MW}]$ is gotten by effectively generation brake.

Table 1: $\quad$ Power consumption.

\begin{tabular}{|c|c|lc|}
\hline $\begin{array}{l}\text { Running } \\
\text { time }[\mathrm{sec}]\end{array}$ & $\begin{array}{l}\text { Power consumption } \\
\text { amount }[\mathrm{MWs}]\end{array}$ & $\begin{array}{l}\text { Maximal moment power } \\
\text { consumption [MW] }\end{array}$ \\
\hline 550 & 366.2 & 4.8 \\
\hline
\end{tabular}

Weak points of this method are the need for a huge memory on PC and a very long computation time.

\section{Conclusion}

In this paper, to reduce power consumption in railways, we presented the D.P. model to solve running curves considering generation brakes, and we showed a schedule of train diagrams to minimize consumption on sample lines.

In the model, we prepared distance and requirement time between stations for the trains, calculated running curves in dimension of number of trains using D.P., and created an $\mathrm{S}$ curve of train diagrams. The evaluation values are both for total and for moment power consumption. The solution is a combination of how to operate trains in order to minimize the values. In principle, this method can be applied in the case of long distance and with many trains.

This method, when applied to two trains on a short line, indicates that the generation brake is used effectively in the solution when one train accelerates and the other one decelerates at the same moment.

Problems that need to be adressed in a future study are the following:

- arrangement to calculation evaluation value by total and moment power consumption.

- improvement algorithm to shorten the computation time

- reduction of PC memory needed

- correspondence for longer distance 
- additional train numbers

- consideration of gradient and limited velocity.

\section{Acknowledgement}

Special thanks go to Mr. Yuichi Nemoto, a graduate student, who programmed part of this method.

\section{Reference}

[1] T.Katori, Y.Nemoto and T.Izumi, "Trains operation time to improve power consumption”, JISAC'07, 3-55 (Aug/2007) (in Japanese)

[2] T.Ibaraki, "Algorithms and Data Structures in C", Syokodo (1999) (in Japanese)

[3] K.Kamahara, "Capacity calculation methods of power facilities", Japan rail electric technology (1999) (in Japanese) 